
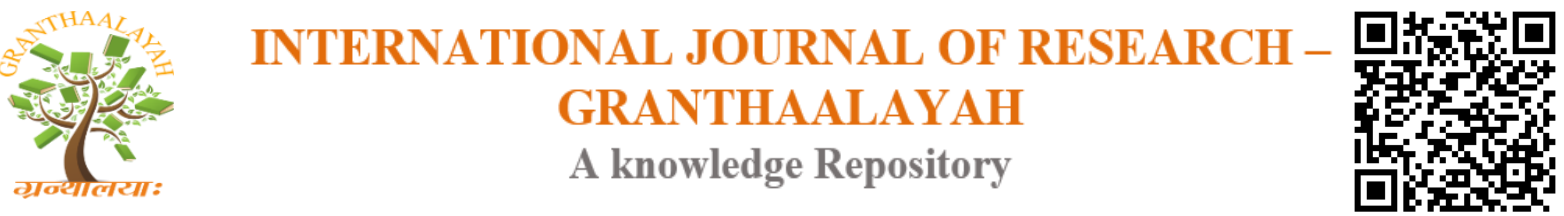

Science

\title{
CLONING AND EXPRESSION OF Lb-PROTEASE FROM cDNA CLONE OF FOOT-AND- MOUTH DISEASE VIRUS
}

\author{
Swaroop Sarkar $^{* 1}$, V.V.S.Suryanarayana ${ }^{2}$, S.R.Madhan Shankar ${ }^{3}$ \\ ${ }^{* 1}$ Research and Development Centre, Bharathiar University, Coimbatore- 641046, India \& \\ Indian Veterinary Research Institute, Hebbal, Bangalore - 560024, India \\ ${ }^{2}$ Indian Veterinary Research Institute, Hebbal, Bangalore - 560024, India \\ ${ }^{3}$ PSG College of arts and science, Coimbatore, Tamil Nadu- 641046, India
}

\begin{abstract}
Foot-and -Mouth disease virus (FMDV) is a positive sense RNA virus and the genome codes for single polyprotein. The FMDV L protein is located at the $\mathrm{N}$ terminus of the polyprotein and is the first gene product released from the nascent polyprotein. The leader L protease which is involved in pathogenesis has two known functions: (i) auto-catalytic removal from the $\mathrm{N}$ terminus of the viral polyprotein and (ii) cleavage of the p220 subunit of the eukaryotic initiation factor $4 \mathrm{~F}$ complex, which helps to shut off host protein synthesis. To explore the role of $\mathrm{L}$ protease in FMDV pathogenesis we generated synthetic FMDV genome lacking the L gene. The gene was amplified from an infectious cDNA clone of serotype Asia1. Primers corresponding to L protease were designed based on the sequence available in the data base. An amplified DNA of $546 \mathrm{bp}$ was purified and cloned into pET28 cloning vector. The sequence analysis revealed the presence of single Open Reading Frame (ORF) encoding a protein of 173 amino acid residues. The sequence alignment using BLAST search in NCBI gene Bank showed $91 \%$ homology with FMDV strain A isolate IND17/77 L protease gene. The recombinant plasmids pETLb was transferred into BL21 (DE3) pLysS cells and the IPTG induced expressed protein of $25 \mathrm{KDa}$ was purified by nickel affinity column as per the manufacturer's protocol (Sigma, USA). The specificity of the expressed protein in was confirmed by western blotting using convalescent cattle serum/ rabbit anti-bovine horse radish peroxidase conjugate and O-Dianisidine Dihydrochloride substrate.
\end{abstract}

Keywords: Cloning; Expression; FMDV Virulence Factor; Lb-Protease.

Core Tip: To explore the role of L protease in FMDV pathogenesis we generated synthetic FMDV Asia 1 lacking the $\mathrm{L}$ gene. Partial L protease (Lb) gene was amplified from the cDNA clone of Asia I 63/72 (Indian Vaccine Strain), subcloned and studied.

Cite This Article: Swaroop Sarkar, V.V.S.Suryanarayana, and S.R.Madhan Shankar. (2017). "CLONING AND EXPRESSION OF Lb-PROTEASE FROM cDNA CLONE OF FOOT-ANDMOUTH DISEASE VIRUS.” International Journal of Research - Granthaalayah, 5(9:SE), 6271. https://doi.org/10.5281/zenodo.1020885. 


\section{Introduction}

Foot-and-mouth disease (FMD) is one of the most contagious diseases of cloven hoofed animals having high economic impact. The causative agent, a virus belongs to the genus Apthovirus, family picornaviridae[1]. FMD is an economically devastating disease of livestock and the presence of the virus in part of the globe is a threat to rest of the world. Although mortality is usually low, morbidity can reach $100 \%$ causing severe losses in production. Therefore, the disease remains a major concern to many developing countries and a continued threat to disease free countries [2,3]. Millions of animals are sacrificed every year worldwide under FMDV eradication programmes [4]. FMDV has continuously circulated ever since after the first outbreak in America in 1870 [5,6]. Further, new serotypes are continuously evolving due to an infinite mutation rate in the RNA genome of the virus [7]. Currently vaccination in India is carried out using trivalent inactivated viral vaccines but there are several limitations associated with inactivated vaccines, major of which is the release of live virus either while handling or through the vaccine, propagation of virulent virus, limited shelf-life and booster injection requirement after 4-12 months [8]. Under this situation production of attenuated virus based vaccine may be safe and ideal. Attenuated virus vaccines are time tested for having proven their worth in eradicating many diseases. However, being highly mutable, naturally selected FMDVs are not considered as reliable vaccine candidates. With the availability of advanced genetic engineering techniques it is now possible to delete any gene segment and make the virus replication defective. Such viruses are ideal candidates for development of attenuated virus vaccines. The FMDV genome contains a single open reading frame of about 7000 bases in length that is initiated from an internal ribosome entry site (IRES) about 1000 bases from the 5' end of the RNA. The Leader (L) appears to cleave at only a single site in the poly protein. The L gene is positioned at the 5' end of the open reading frame and contains two potential in-frame initiation codon(84 nucleotides apart). The L-Protease is responsible for pathogenicity by inhibiting the host protein synthesis. L-Protease mutants have been found to reduce pathogenicity and replication efficiency of the virus. Moreover the deletion helps in developing marker vaccine. Here we report the cloning, expression and characterization of L-Protease. The gene is expressed in E.coli and the protein expressed is used to raise antiserum in rabbit which could be further used to develop companion tests for evaluating marker facility of the gene deleted attenuated virus.

\section{Report}

\section{Gene, Host strain and Plasmid Vectors:}

Foot and mouth disease virus serotype Asia 1 63/72 infective cDNA clone available in the laboratory (Suryanarayana et al 2003) was used for amplifying Lb-Protease gene. Commercially available E.coli host strains DH5 $\alpha$ and BL21 DE3 p(Lys)S and expression vector pET 28 A were used for L-protease expression.

\section{Lb protease amplification Cloning:}

The Lb-Protease sequence was amplified from the full length infective cDNA using the primers LPro (L) 5' GCG CCA TAT GGA ATT CAC ACT CTG CAA CGG T as forward and L-Pro (R ) GCG CTC GAG TGC GGC CGC CTT GAG TCG TTT CTG AAC CTT as reverse primers. These primers were designed based on the published sequence (Saravanan et al 2003). A 50 $\mu 1$ 
reaction mix containing $1.5 \mathrm{mM} \mathrm{Mgcl}_{2}, 100 \mu \mathrm{M}$ each of dNTPS, 25mM Tris-HcL, 20 pmol of each primer and one unit of Taq DNA polymerase was used for amplification in the Thermal Cycler (perkin-Elmer Cetus, USA) with initial denaturation at $95^{\circ} \mathrm{C}$ for $3 \mathrm{~min}$, followed by 35 cycles of $95^{\circ} \mathrm{C}$ for $1 \mathrm{~min}, 60^{\circ} \mathrm{C}$ for $1 \mathrm{~min}$ and $72^{\circ} \mathrm{C}$ for $1 \mathrm{~min}$ with a final extension at $72^{\circ} \mathrm{C}$ for 10min. the amplified PCR product corresponding to Lb-Protease (500bp) gene was purified using wizard PCR prep DNA purification system (Promega, USA), digested with Nde1 and Xho1 and the digested product was ligated into pET28 at Nde1 and Xho1 sites. The transformants were selected on kanamycin plates. The transformants were initially screened by colony PCR, using vector specific T7 promoter sequence primer and insert specific reverse primer to select the recombinants, followed by insert release from the recombinant plasmid DNA.

\section{Sequencing of Lb-Pro Gene:}

The L-Pro gene in the plasmid pETLb was sequenced using T7 promoter and Lpro(R) primers in ABI 377 automated DNA sequencer (ABI inc., USA). Sequence data obtained was analyzed by aligning with published FMDV L-protease gene sequences available in NCBI data base. Both nucleotide and derived amino acid sequence were compared with the published Asia 1 sequence.

\section{Expression and purification of cloned Lb-Pro:}

The recombinant plasmids pETLb was transferred into BL21 (DE3) pLysS cells. Three individual clones were selected and grown seperately in $10 \mathrm{ml}$ LB broth with Kanamycin $(50 \mu \mathrm{g} / \mathrm{ml})$ for overnight at $37^{\circ} \mathrm{C}$ with shaking. One hundred microliter of the overnight culture was inoculated into fresh $10 \mathrm{~mL} \mathrm{LB}$ broth without antibiotic for $3 \mathrm{~h}$ at $37^{\circ} \mathrm{C}$. The protein expression was induced by adding IPTG (isopropyl-beta-D-thiogalactopyranoside) to a final concentration of $1 \mathrm{mM}$ at $30^{\circ} \mathrm{C}$ for $6 \mathrm{~h}$ and the cells were pelleted and resuspended in $1 \mathrm{~mL}$ of TE containing 10mMphenyl methyl sulfonyl fluoride ( PMSF) ( $\mathrm{pH} \mathrm{8.0)}$ and stored at $80^{\circ} \mathrm{C}$ for further work. The expressed protein in the cell lysate was purified by nickel affinity column as per the manufacturer's protocol (Sigma, USA). The proteins in the cell lysate were diluted 4 folds with column loading buffer ( $8 \mathrm{M}$ urea in $10 \mathrm{mM}$ Tris HCl, 1mM EDTA, pH.8.0) and centrifuged at $15000 \mathrm{xg}$ at $4^{0} \mathrm{C}$ to remove any debris. The solution was concentrated by polyethylene glycol, dialysed against phosphate buffered saline (PBS) and stored at $-70^{\circ} \mathrm{C}$. The purified protein was separated on $12 \%$ polyacrylamide gel under denaturing conditions as per the mothod of Laemmli.

An aliqot $(20 \mathrm{ul})$ of the cell lysate was analysed by $10 \%$ Sodium Dodecyl SulpfatePolyacrylaminde Gel Electrophoresis (SDS-PAGE) as per standard protocol in comparison with the same quantity of lysates from vector transformed cells and uninduced cloned cells. The specificity of the expressed protein in was confirmed by western blotting using, the convalescent bovine serum as primary antibody, and antibovine antibody horse radish peroxidase conjugate as secondary antibody and O-Dianisidine Dihydrochloride as substrate as per standard protocol (Towban)

A similar duplicate gel was blotted onto a PVDF membrane for immune detection. The recombinant protein was detected by treating with anti-convalescent serum (at 1:1000 dilution), followed by an anti-rabbit antibody HRPO conjugate (1:1000) and orthodianisidine dihydrochloride (ODD) as substrate. 


\section{Results and Discussion}

The amplified PCR product corresponding to L-Protease b (Lb) of size 546 nts.(519 gene size + 27nts (Fig.1) was purified using wizard PCR prep DNA purification system (Fementas, USA) and cloned into Nde1 and Xho1 sites in frame in pET28a vector.

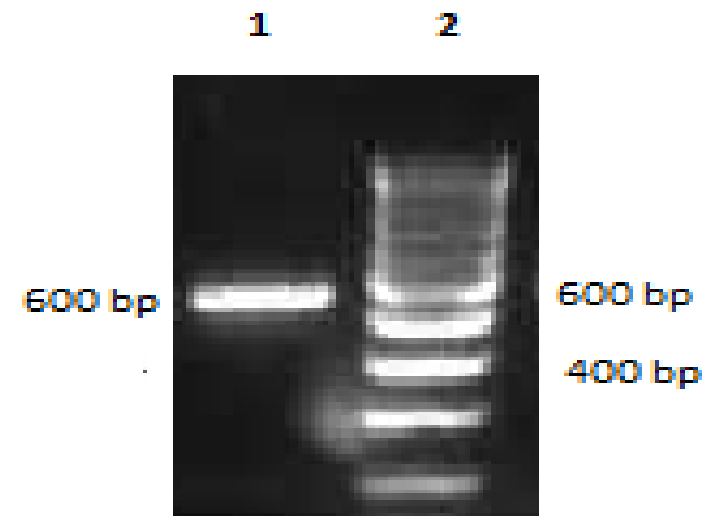

Figure 1: PCR amplification of Lb protease gene

\section{Lane 1, PCR amplified Lb protease; Lane 2, 100 bp ladder}

Several Kanamycin resistant colonies were observed on LB -agar-Kanamycin plate (Fig not shown). They were screened by PCR and a few of the PCR positive clones were selected, plasmid purified and the presence of insert corresponding to Lb-Protease gene in pET28a vector was confirmed by restriction digestion with Nde 1 and Xho 1 and analysis by agarose gel electrophoresis using standard molecular size markers. As seen in the Fig 2. an insert of around $600 \mathrm{bp}$ was released from one of the recombinant plasmids (Fig.2, lane 1)

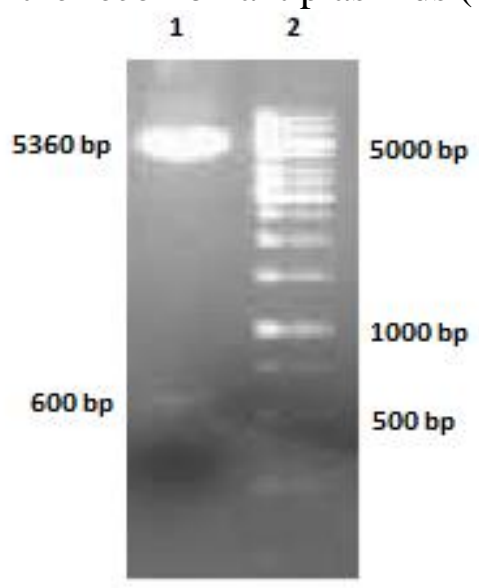

Figure 2: R.E anlysis of pET 28 plasmid with Lb-protease gene

\section{Lane 1, Nde 1-Xho 1 digested recombinant plasmid; Lane 2, 1.0kb DNA ladder}

The cloned insert was subjected to nucleotide sequence using T7 promoter primer as described under Methods. A sequence of 743 nts was read from the ladder sequence from 5' end which includes vector sequences (Fig. 3). When the nucleotide sequence was translated using on-line programme an aa sequence of 173 aa (Fig 4) was obtained with no stop codon in between. Identity of the cloned sequence was confirmed by Nucleotide Blast search using NCBI online programme of sequence analysis (Fig.5) 
CCATGGGCAGCAGC CATCATCATCATCATCACAGCAGCGGCCTGGTGCCGCGCG GCAGCCATATGGCTAGCATGACTGGTGGACAGCAAATGGGTCGCGGATCCGAA TTCACACTCTGCAACGGTGAGAAGAAGACCTTCTACTCCAGGCCCAACAACCACGA CAACTGTTGGTTGAACACCATCCCCCAGTTGTTTAGGTACGTCGATGAACCTTTCTTC GACTGGGTCTATGAATCACCTGAGAACCTCACTCTTGAGGCGATCAAACAATTGGAA GAGGTTACTGGTCTTGAACTGCACGAGGGTGGGCCACCCGCTCTCGTCATCTGGAAC ATCAAGCACTTGCTTCACACTGGAATCGGTACCGCCTCGCGACCAAGCGAAGTGTGT ATGGTGGACGGCACGGACATGTGCCTGGCTGACTTCCACGCTGGCATTTTCCTGAAA GGACAGGAACACGCTGTGTTTGCCTGTGTCACCTCCAACGGGTGGTACGCGATTGAC GACGAGGACTTTTACCCCTGGACGCCGGACCCGTCCGATGTCCTGGTATATGTTCCG TACGATCAAGAACCACTCAACGGAGAACGGAAAGCAAAGGTTCAGAAACGACTCA AGGCGGCCGCACTCGAGCACCACCACCACCACCACTGAGATCCGGCTGCTAACA AAGCCCGAAAGGAAGCTGAGTTGGCTGCTGCCACCGCTGAGCAATAACTAGCA TAACCCCTTGGGGCCTCT

Figure 3: Nucleotide sequence of the cloned Lb -protease gene

Vector sequences are underlined. His tag sequences at 5' and 3' ends from the vector are shown with italics. ATG codons are shown with bold letters.

MEFTLCNGEKKTFYSRPNNHDNCWLNTIPQLFRY VDEPFFDWVYESPENLTLEAIKQLE E 60

VTGLELHEGGPPALVIWNIKHLLHTGIGTASRPSEVCMVDGTDMCLADFHAGIFLKGQE H 120

AVFACVTSNGWYAIDDEDFYPWTPDPSDVLVYVPYDQEPLNGERKAKVQKRLK 173

Figure 4: Derived amino acid sequence of the cloned Lb protease gene

Foot-and-mouth disease virus - type A isolate IND17/77 L protease gene, partial cds Sequence ID: gb|FJ265694.1|Length: 603Number of Matches: 1

Range 1: 88 to 603 GenBankGraphicsNext MatchPrevious Match
699 bits(378)
$0.0 \quad 470 / 516(91 \%)$
$0 / 516(0 \%)$
Plus/Plus

Query 1

GAATTCACACTCTGCAACGGTGAGAAGAAGACCTTCTACTCCAGGCCCAACAACCA CGAC 60

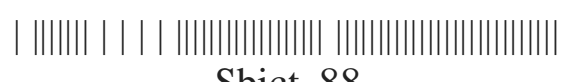

Sbjet 88

GAATTCACACTTTACAACGGTGAGAAGAAGACTTTCTACTCCAGGCCCAACAACCA CGAC 147

Query 61

AACTGTTGGTTGAACACCATCCCCCAGTTGTTTAGGTACGTCGATGAACCTTTCTTCG AC 120

||||||||| |||||||||||| |||||||||||||| ||||||||||||||||||||| 
Sbjet 148

AACTGTTGGCTGAACACCATCCTTCAGTTGTTTAGGTATGTCGATGAACCTTTCTTCG AC 207

Query 121

TGGGTCTATGAATCACCTGAGAACCTCACTCTTGAGGCGATCAAACAATTGGAAGA GGTT 180

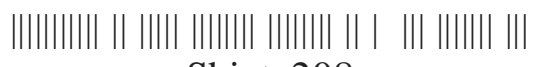

Sbjet 208

TGGGTCTATGACTCGCCTGAAAACCTCACGCTTGAGGCCATAAGGCAACTGGAAGA AGTT 267

Query 181

ACTGGTCTTGAACTGCACGAGGGTGGGCCACCCGCTCTCGTCATCTGGAACATCAAG CAC 240

|||||||||||||| ||||||||||| || |||||||||||||||||||||||||| |||

Sbjet 268

ACTGGTCTTGAACTACACGAGGGTGGACCGCCCGCTCTCGTCATCTGGAACATCAAA CAC 327 Query 241

TTGCTTCACACTGGAATCGGTACCGCCTCGCGACCAAGCGAAGTGTGTATGGTGGAC GGC 300

Sbjet 328

CTTCTCCACACCGGAATCGGCACTGCCTCGCGCCCCAGCGAGGTGTGTATGGTGGAC GGA 387 Query 301

ACGGACATGTGCCTGGCTGACTTCCACGCTGGCATTTTCCTGAAAGGACAGGAACAC GCT 360 ||||||||||| ||||||||||||||||||||||||||||||||||||| || || |||

Sbjet 388

ACGGACATGTGTTTGGCTGACTTCCACGCTGGCATTTTCCTGAAAGGACAAGAGCAT GCT 447 Query 361

GTGTTTGCCTGTGTCACCTCCAACGGGTGGTACGCGATTGACGACGAGGACTTTTAC CCC 420

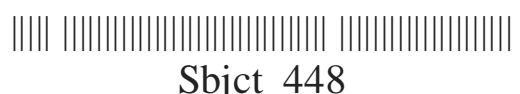

GTGTTCGCCTGTGTCACCTCCAACGGGTGGTACGCGATCGACGACGAGGACTTTTAC CCC 507 Query 421

TGGACGCCGGACCCGTCCGATGTCCTGGTATATGTTCCGTACGATCAAGAACCACTC AAC 480 ||||| |||||||||||||| |||||||||| |||||||||||||||||||||||| ||| Sbjct 508

TGGACACCGGACCCGTCCGACGTCCTGGTATTTGTTCCGTACGATCAAGAACCACTT AAC 567

Query 481 GGAGAACGGAAAGCAAAGGTTCAGAAACGACTCAAG 516 


\section{|||||| |||| |||||||||| || |||||||||}

\section{Sbjct 568 GGAGAATGGAAGGCAAAGGTTCAAAAGCGACTCAAG 603}

Figure 5: Sequence alignment of the ladder sequence using BLAST search programme

The BLAST search (Fig 5) showed the presence of FMDV L protease gene sequence devoid of 87 nucleotides corresponding to 29 aa at $\mathrm{N}$-terminal end, termed as Lb protease. As shown in Fig.5, when the ladder sequence was aligned with the L-protease gene of FMDV 'A' the alignment shows that the protein has a homology of $91 \%$ with the published L-protease gene sequence. The second ATG codon is in frame with the rest of the sequence indicating the intactness of the second coding region which is reported to be in-frame with the fist ATG codon. The protein derived from second ATG codon is designated as Lb while entire protein is designated as Lab. As per the literature both are active proteins and can function Cis-active proteases, which are released post translational from poly protein at $\mathrm{N}$-terminal end of the FMDV poly-protein of $250 \mathrm{kDa}$. Preference for initiation codon depends on $\mathrm{Mn}^{2+}$ concentration. The recombinant $\mathrm{pET}$ Lb was transferred into BL-21 DE3 pLys cells. Three of the several transformants were selected, grown in LB broth in the presence of Kanamycin and induced with IPTG for expression of the cloned gene and the lysate proteins were analyzed by $10 \%$ SDS SAGE. The gel was stained with Comassie Brilliant Blue (CBB) and de-stained as described under materials and methods (Fig 6). As seen in the Fig 6 Several protein bands were seen in the CBB stained gel in the case of both induced and un-induced cells (lane 1-6) which may be host or vector specific. However, a single intense protein band of size around $25 \mathrm{kDa}$ is visible only in the case of induced cultures carrying vector with Lb gene as insert (lane 1-3) but not in the case of un-induced (lane 4,5) and vector transformed and induced culture (lane 6) indicating that $25 \mathrm{kDa}$ protein corresponds to the cloned Lb protein gene. The other proteins may be of host or vector specific.

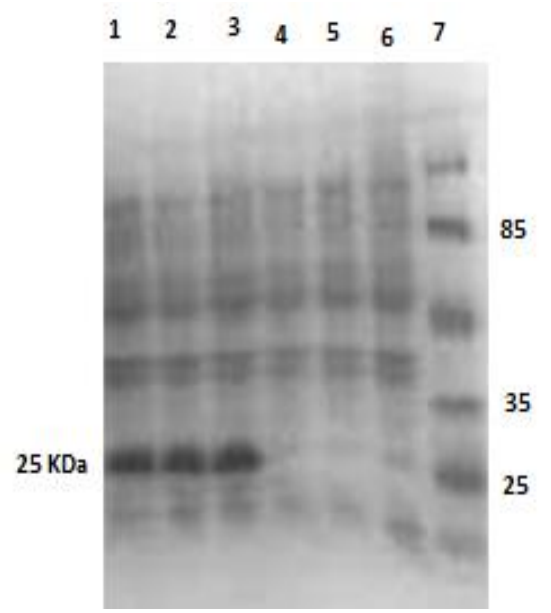

Figure 6: SDS PAGE anlysis of E.coli expressed proteins from pET 28 vector with or without interest

Lane 1,2,3. Proteins from induced clones $(1,2,3)$; Lane 4,5 proteins from uninduced clines $(1,2)$; Lane 6, protein from vector transformed induced E.coli cells ; 7 Protein molecular weight markers

Since, the cloned gene sequence is in frame with the ATG codon of pET28a the induction with IPTG has result in the expression of the gene to produce a fusion recombinant protein (Fig 6) 
carring N-Terminal (20aa) and c-terminal (13aa) fusion that has all the Tags including His Tag. By inserting the gene lacking termination codon at 3' end, the His Tag at the N-terminus of the vector is also in frame. Therefore the total coding region excluding Tag protein comprises 645 nucleotides i.e 215 amino acids corresponding to $\sim 24 \mathrm{KDa}$. High intensity protein bands revealed that the protein was over expressed and present in soluble form.

The protein corresponding to Lb-Protease as per the sequence was expected to have molecular weight of $19 \mathrm{KDa}$. However the observed molecular weight of $24 \mathrm{KDa}$ is due to the presence of tags at Nand Ctreminal regions. Presence of His-tag at amino terminus of expressed proteins from pET28a vector had enabled us to purify the recombinant protein from E.coli using HISselect nickel affinity gel column (Fig.7). The specificity of the expressed protein was confirmed by Western Blot assay where antiserum from convalescent bovine was used. The intense positive colour reaction shows the specificity (Fig.8). The background colour reactions seen above and below the specific protein may be due to the presence of other E.coli proteins which have co purified as contaminants that were not visible in the CBB stained gel (Fig 8, lane 1).

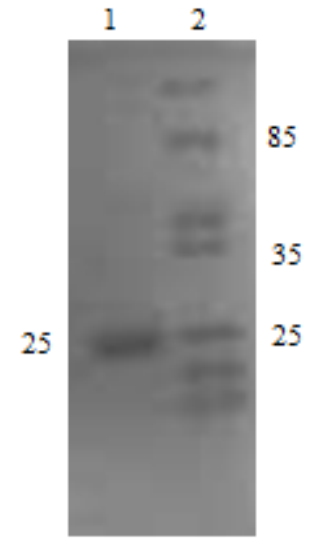

Figure 7: SDS PAGE of the affinity purified Lb protein expressed in E.coli

\section{Lane 1, affinity purified Lb fusion protein; Lane 2, Protein molecular weight markers}

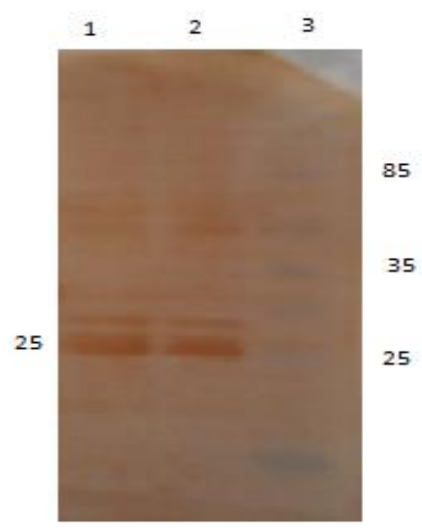

Figure 8: Western Blot analysis of E.coli expressed Lb fusion protein

Lane 1,2 : Affnity purified expressed protein in fraction 1 and 2 respectively; lane 3, Prestained protein molecular weight markers.

Among the picornaviruses, only members of apthovirus genus contain an active leader (L) proteinase gene. The L proteinase has only two known fuctions, its autocatalytic removal from 
the $\mathrm{N}$ terminus of the viral polyprotein and cleavage of the p220 subunit of eIF-4F. Cleavage of p220 is thought to be responsible for inhibiting the translation of capped host cell mRNAs in the presence of viral translation which occurs by a cap-independent mechanism. Cleavage of p220 appears to be an important for picornavirus replication, since the rhinoviruses and enteroviruses utilize a different proteinase (2A) to cleave p220.

The major objective of producing L protease is to raise antiserum against L-Pro and utilize the same for the detection of attenuated leaderless virus developed by us. The L-proteinase gene of FMDV was amplified and sequenced. The complete L proteinase gene could be expressed in soluble form with six histidines which was used as a tag to purify the soluble protein expressed in E.coli. The specificity of the expressed protein was confirmed by Western Blot assay blot assay where antiserum against Lb-Protease (purified E.coli expressed protein) raised in rabbits was used. The calculated size of the L-Protease comes to $24 \mathrm{KDa}$ which is in agreement with the protein size observed in SDS-PAGE. The protein could be used as diagnostic antigen for the development of companion test to be used in L-deleted attenuated FMDV vaccine.

\section{Acknowledgement}

The financial assistance from the department of Indian Council of Agricultural Research, Pusa, New Delhi, is gratefully acknowledged. We thank the Director, Indian Veterinary Research (IVRI), Izatnagar, Bareilly and the Joint Director, IVRI, Bangalore Campus, India, for providing the facility to carry out this work.

Author's Contribution: V.V.S.Suryanarayana designed the report and planned the work; Swaroop Sarkar performed the bench work; V.V.S.Suryanarayana and S.R.Madhan Shankar analysed the data; Swaroop sarkar and V.V.S.Suryanarayana wrote the paper.

Supported by: Indian Council of Agricultural Research (ICAR), New Delhi, Director, Indian Veterinary Research (IVRI), Izatnagar, Bareilly and Joint Director, IVRI, Bangalore Campus, India

Institutional Review Board Statement: The study was reviewed by ICAR and Staff Research Council (SRC) of IVRI, Bangalore.

Conflict-Of-Interest Statement: V.V.S.Suryanarayana has received research funding for this project under Emeritus Scientist Scheme from ICAR, New Delhi. Swaroop Sarkar was a Senior Research Fellow worked under the project at Indian Veterinary Research Institute, Hebbal, Bangalore.

Correspondence to: V.V.S.Suryanarayana (Emeritus Scientist), Indian Veterinary Research Institute, Hebbal, Bangalore - 560024, India. veluvarthy@yahoo.com

Telephone: +91-9449341844

\section{References}

[1] Francisco S,Margarita S,Miguel A J,etal.Foot-and-Mouth disease virus:along known virus, but a current threat [J]. Vet res, 2001,32:12-30 
[2] Knlowles, N.J. and Samuel, A.R. (2003) molecular epidemiology of foot and mouth disease virus. Virus res, 91(1):65-80

[3] Ko, Y.J., Jeoung, H.Y., Lee, H.S., Chang, B.S., Hong, S.M., Heo, E.J., Lee, K.N., Joo, H.D., Kim, S.M., Park,J.H. and Kweon,C.H.(2009) A recombinant protein-based ELISA for detecting antibodies to foot-and-mouth disease virus serotype Asia1. J.Virol.Methods, 159(1):112-118.

[4] Rodriguez LL, Grubman MJ. Foot and mouth disease virus vaccines. Vaccine. 2009;4(Supplement 4):D90-4.

[5] Gibbs P. The foot-and-mouth disease epidemic of 2001 in the UK: implications for the USA and the "war on terror". J Vet Med Educ. 2003;30:121-32.

[6] Sumption K, Rweyemamu M, Wint W. Incidence and distribution of footand-mouth disease in Asia, Africa and South America; combining expert opinion, official disease information and livestock populations to assist risk assessment. Transbound Emerg Dis. 2008;55:5-13.

[7] Anonymous. Foot-and-mouth disease. In: Manual of diagnostic tests and vaccines for terrestrial animals (mammals, birds and bees). Paris, France: World Organization for Animal Health (OIE); 2008.

[8] Rodriguez LL, Gay CG. Development of vaccines toward the global control and eradication of foot-and-mouth disease. Expert Rev Vaccines. 2011;10:377-87

[9] T.sarvanan, G.R.Reddy, H.J.Dechamma, V.V.S.Suryanarayana.(2003) Construction of gnomelength cDNA for foot-and-mouth disease virus serotype Asia 1 IND 63/72 vaccine strain.IJBMBR,2(2).

[10] Bradford M M, A rapid and sensitive method for the quantification of microgram quantities of protein utilizing the principle of protein-dye binding, Anal Biochem, 72 (1976) 248-254.

[11] Laemmli U K, Cleavage of structural protein during the analysis of head of bacteriophage T4, Nature (Lond), 227 (1970) 680-685.

[12] Brown, C. C., M. E., Piccone, P.W.Mason, T.S.C. Mckenna, and M.J.Grubman. 1996. Pathogenesis of wild-type and leaderless foot-and-mouth disease virus in cattle. J.Virol. 70:56385641 . 